\title{
THE EFFECT OF CONTACT ANGLE ON DYNAMICS OF DRY SPOTS SPREADING IN A HORIZONTAL LAYER OF LIQUID AT LOCAL HEATING
}

\author{
Zaitsev D.V. ${ }^{1,2, a}$, Kirichenko D.P. ${ }^{1,3}$, Orlik E.V. ${ }^{2}$, Kabov O.A. ${ }^{1,2}$ \\ ${ }^{1}$ Kutateladze Institute of Thermophysics SB RAS, 630090 Novosibirsk, Russia \\ ${ }^{2}$ National Research Tomsk Polytechnic University, 634050 Tomsk, Russia \\ ${ }^{3}$ Novosibirsk State University, 630000 Novosibirsk, Russia ${ }^{a}$
}

\begin{abstract}
The effect of equilibrium contact angle on dynamics of dry spot spreading at disruption of a horizontal water layer heated locally from the substrate was studied using the high-speed Schlieren technique. Different methods of working surface processing were applied; this allowed variations of the equilibrium contact angle from $27 \pm 6^{\circ}$ to $74 \pm 9^{\circ}$ without a change in thermal properties of the system. It is found out that substrate wettability significantly affects the propagation velocity of dry spot and its final size. It is also found out that the velocity of contact line propagation is higher in the areas of substrate with a higher temperature.
\end{abstract}

\section{Introduction}

The physical processes in the thin layers (films) of liquid are widely used in industry, as they provide high intensity of heat and mass transfer, but the thin liquid films are subjected to rupture, which drastically reduces the effectiveness of devices and can lead to their failure. On the other hand, a submicron liquid film formed on the border of the washable dry spots can make a significant contribution to overall heat and mass transfer due to intense evaporation [1].

An important aspect of the problem of liquid film rupture is the question about the influence of substrate wettability [2]. The complexity of experimental study of the wettability influence is caused by the fact that with a change in liquid or substrate material not only the contact angle changes, but also the thermal properties of the system, which can significantly affect the film rupture.

The aim of this study is to investigate the influence of equilibrium contact angle on dynamics of dry spot spreading at disruption of a horizontal, locally heated thin layer of water. One substrate is used in the current study, but with different surface treatment that allows to vary the equilibrium contact angle within a wide range without changing the thermal physical properties of the system.

\section{Experimental setup and methods}

The experimental scheme is shown in Fig. 1. The basis of the test section is a stainless steel plate of $3 \times 50 \times 150 \mathrm{~mm}^{3}$ with a copper rod of $10 \times 10 \mathrm{~mm}^{2}$ cross-section, embedded flush, which serves as a

${ }^{\text {a }}$ Corresponding author : zaitsev@itp.nsc.ru 
heating element. The heater surface temperature, $T_{w}$, and surface temperature of stainless steel plate are determined at several points by thermocouples. The source of heat is a nichrome spiral wound on the free part of the rod. A layer of insulation material (mineral wool) is superimposed over the spiral. The heat flux, $q$, is determined by the electric power released at the spiral. Degassed distilled water with the initial temperature of $25^{\circ} \mathrm{C}$ is used as the working liquid. The test section is open to the atmosphere; the temperature of ambient air is $23 \pm 2^{\circ} \mathrm{C}$. The test section is installed horizontally, and a square cooper cooling circuit is mounted on the working surface, so that the heater is in its center. The inner size of the circuit is $40 \times 40 \mathrm{~mm}^{2}$. Water with the temperature of $25^{\circ} \mathrm{C}$ is pumped through the circuit. Then, a given volume of working liquid is put on the working surface by means of a syringe. In the experiment, the initial thickness of liquid layer, $h_{0}$, varies from 0.40 to $0.60 \mathrm{~mm}$.

To register dynamics of dry spot spreading, the Schlieren method [3] with the high-speed camera FASTCAM SA1.1 (5400 frames per second, resolution of $1024 \times 1024$ pixel, optic resolution of up to 10 micron/pixel) was used, Fig. 1.

The equilibrium contact angle on the working surface at different points was determined by the method of a sessile drop at the room temperature of $23 \pm 2{ }^{\circ} \mathrm{C}$. To obtain the profile of the drop surface, the shadow method was used with a collimated light source and digital camera Nikon D800 with resolution of $6 \mathrm{micron} / \mathrm{pixel}$ (see Fig. 1, when measuring the contact angle, the cooling circuit was removed). The resulting images were processed using the software by KRUSS with the accuracy of contact angle measurement of $0.1^{\circ}$. The contact angles measured at different points on the copper heater and surrounding stainless steel surface did not differ within the statistical measurement error.

During the experiment, at the given value of $h_{0}$, the heat flux increased with a small step up to threshold value $q_{i d p}$, when the first dry spot was formed on the heater. In experiments, the maximal heat flux was $32 \mathrm{~W} / \mathrm{cm}^{2}$, and the maximal temperature of the heater surface before layer rupture was $96.5^{\circ} \mathrm{C}$.

Variation in the degree of substrate wettability is achieved by the following method. Before the experiment, the working surface was treated by sandpaper with the grain size of $10 \mu \mathrm{m}$ by wet polishing. At that, the equilibrium contact angle, measured immediately after treatment, was $\theta=27 \pm 6^{\circ}$. After the experiment, the working surface was dried and kept in air for several hours. The equilibrium contact angle increased to $\theta=74 \pm 9^{\circ}$. After that the experiment was repeated. This procedure provided contact angle variation without a change in thermal physical properties of the system and substrate roughness (in each experimental series). The substrate roughness could change from one to another experimental series, but the contact angles were the same within the statistical measurement error.

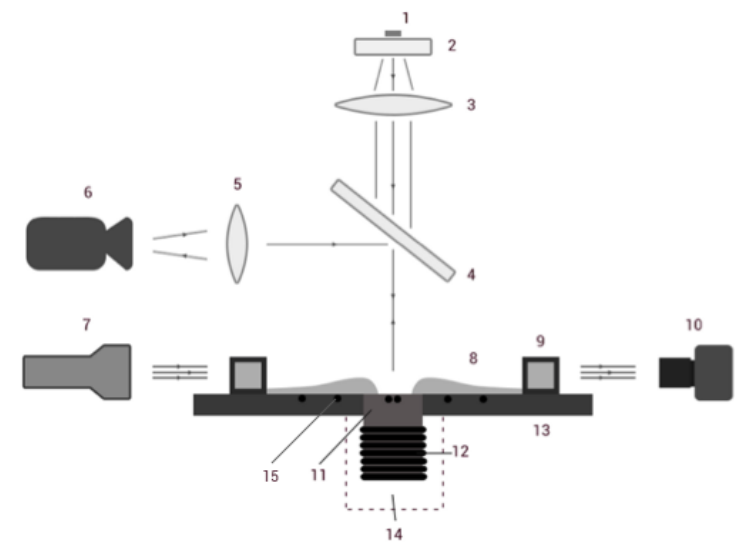

Figure 1. Scheme of experiment. 1 - 6 Schlieren system: 1-light source, 2- opal glass, 3 - collimating lens, 4 beam splitter, 5 - Schlieren lens; 6 - high-speed camera. 7 - source of collimated light; 8 - test section; 9 cooling circuit; 10 - digital camera; 11 - copper rod; 12 - nichrome spiral; 13 - stainless steel plate; 14 - heatinsulating material, 15 - thermocouple. 


\section{Experimental results}

Dynamics of dry spot propagation for the heat flux slightly higher than the threshold value $q_{i d p}$ is shown in Fig. 2 for different contact angles and layer thicknesses. It is seen that a dry spot is always generated near the heater edge, and then it spreads over both the copper heater and surrounding stainless steel surface. The mechanism of layer rupture is thermocapillary because the dry spot is formed at the substrate temperature lower than the saturation temperature $\left(T_{w}<96.5^{\circ} \mathrm{C}\right)$ at the point (near the heater edge), where the temperature gradient on the layer surface is maximal (see the results of thermography study [4]).

The time interval between adjacent photographs in upper row of Fig. $2 \mathrm{a}$ is $0.045 \mathrm{~s}$, while that in lower row is $2.000 \mathrm{~s}$. Thus, one can conclude that the speeds of dry spot spreading for different wetting conditions differ significantly: for $\theta=74 \pm 9^{\circ}$ the speed of spreading is more than 50 times higher than that for $\theta=27 \pm 6^{\circ}$. Besides, it can be seen from Fig. 2 that the final sizes of a dry spot is also very different: for $\theta=74 \pm 9^{\circ}$ the area of the final dry spot is several times higher than that for $\theta=27 \pm 6^{\circ}$.

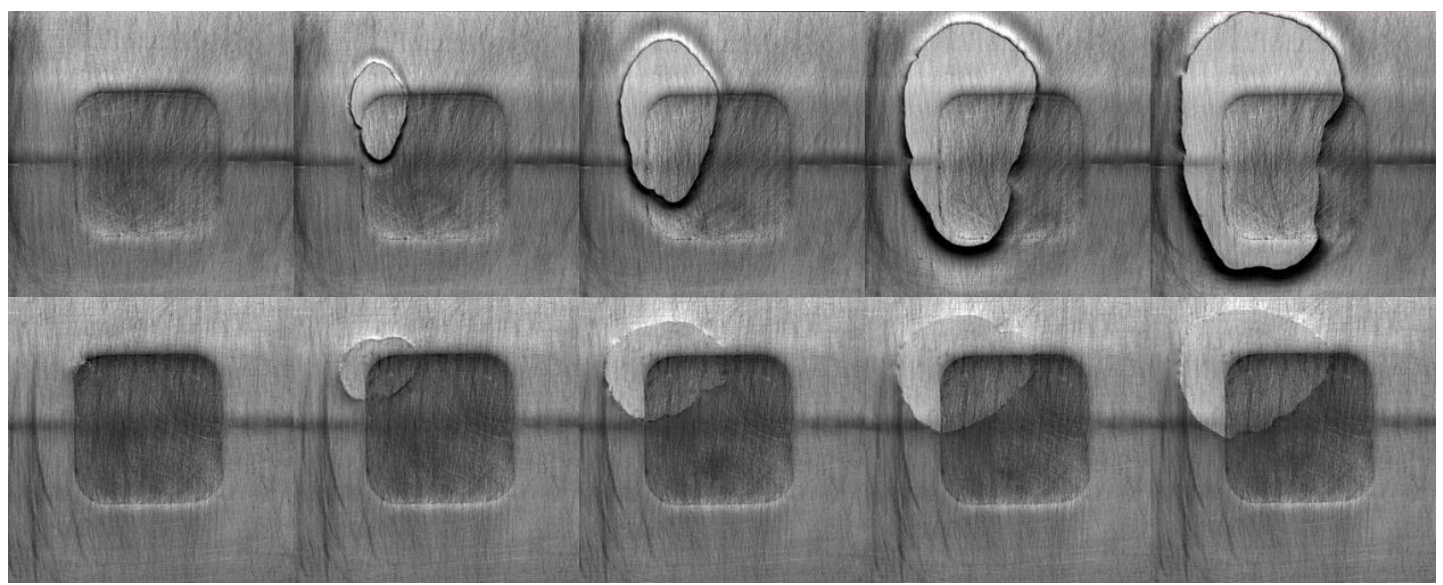

a)

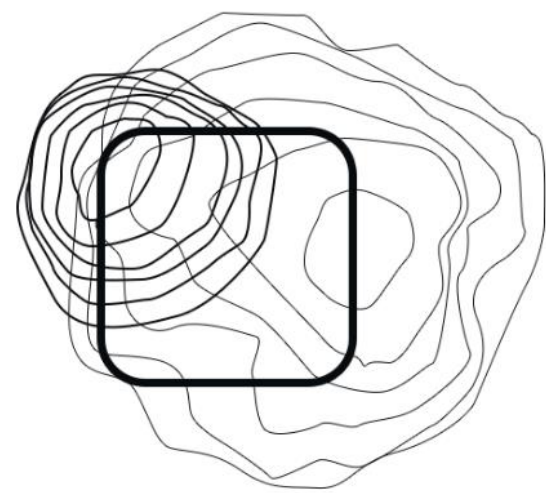

b)

Figure 2. Dynamics of dry spot spreading. a) Schlieren photographs, $h_{0}=0.40 \mathrm{~mm}, q=27.0 \mathrm{~W} / \mathrm{cm}^{2}, T_{w}=85 \pm 3^{\circ} \mathrm{C}$. Upper row of photographs $-\theta=74 \pm 9^{\circ}$, time interval between adjacent photographs is $0.045 \mathrm{~s}$. Lower row of photographs $-\theta=27 \pm 6^{\circ}$, time interval between adjacent photographs is $2.000 \mathrm{~s}$. b) Dry spot contours, $h_{0}=0.60$ $\mathrm{mm}, q=32.0 \mathrm{~W} / \mathrm{cm}^{2}, T_{w}=95 \pm 2^{\circ} \mathrm{C}$. Thin contours are drawn with a step of $0.200 \mathrm{~s}$, and correspond to $\theta=74 \pm 9^{\circ}$. Bold contours are drawn with a step of $2.000 \mathrm{~s}$, and correspond to $\theta=27 \pm 6^{\circ}$. 
The time dependences of the contact line propagation velocity on the copper and stainless steel surfaces for the dry spots, shown in Fig. 2a, are presented in Fig. 3. The velocity was measured along the line passing through the heater edge. It can be seen that the maximal velocity of the contact line propagation for $\theta=74 \pm 9^{\circ}$ (Fig. 3b) reaches $88 \mathrm{~mm} / \mathrm{s}$, which is approximately 80 times higher than the maximal velocity of contact line propagation for $\theta=27 \pm 6^{\circ}$ (Fig. 3a). It can be also seen from Fig. 3 that the velocity of contact line propagation on copper is up to twice as high as the velocity of contact line propagation on stainless steel for both the conditions of good (Fig. 3a) and poor (Fig. 3b) wettability. This is apparently because the temperature of the copper heater surface is significantly higher than that of the surrounding stainless steel surface. As it can be seen from Fig. 4, when the layer ruptures, the heater surface temperature is approximately 30 degrees higher than the temperature of the stainless steel surface near to the heater.

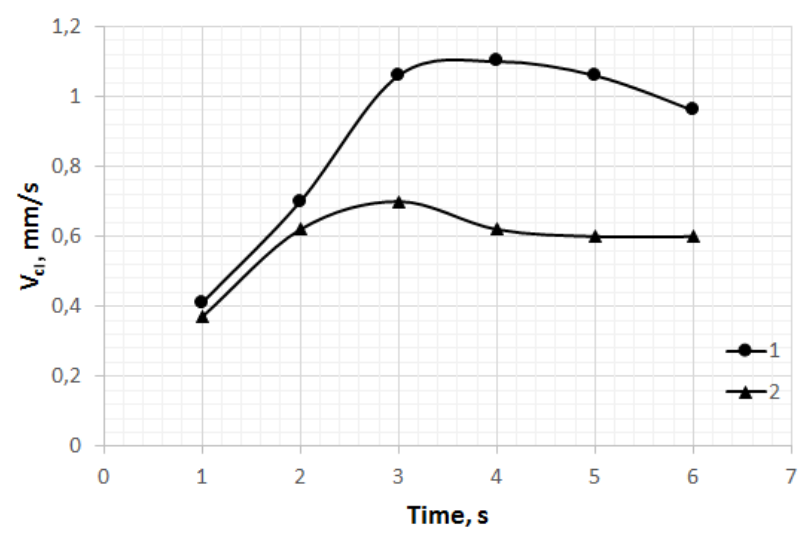

a)

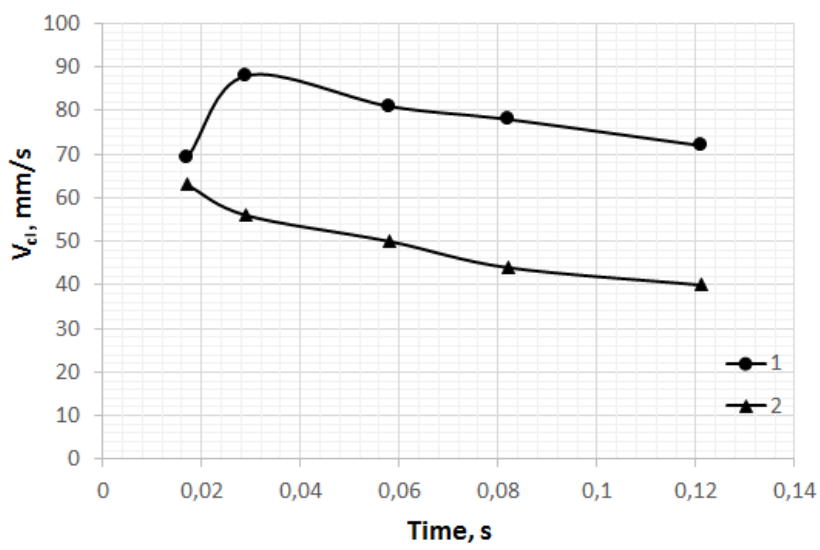

b)

Figure 3. Time dependence of the contact line velocity for dry spots shown in Fig. 2a. a) and b) $-\theta=27 \pm 6^{\circ}$ and $\theta=74 \pm 9^{\circ}$, respectively. 1 and 2 - propagation of contact line on copper and stainless steel, respectively. 


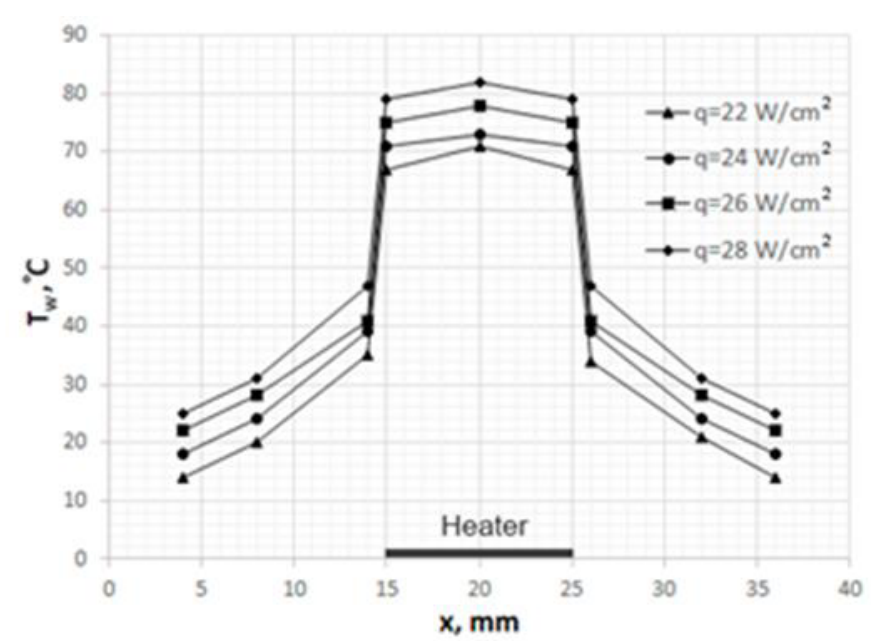

Figure 4. Temperature distribution over the working surface for different heat fluxes, $h_{0}=0.40 \mathrm{~mm}$. At $q=28$ $\mathrm{W} / \mathrm{cm}^{2}$ rupture of the liquid layer occurs; the temperatures are measured immediately after the rupture.

\section{Conclusions}

The influence of the equilibrium contact angle on dynamics of dry spot spreading at disruption of a horizontal thin layer of water, locally heated from the substrate, was studied experimentally. It was determined that:

- The wettability of the substrate affects significantly the velocity of dry spot propagation: for the contact angle of $74 \pm 9^{\circ}$, the velocity of propagation is 2 orders higher than for the contact angle of $27 \pm 6^{\circ}$.

- The wettability also affects the final size of a dry spot: for the contact angle of $74 \pm 9^{\circ}$ the area of the final dry spot is several times larger than for the contact angle of $27 \pm 6^{\circ}$.

- For the conditions of both good and poor wettability, the velocity of contact line propagation is higher in the substrate zones with a higher temperature.

The work was financially supported by Russian Science Foundation (Agreement No. 14-19-01755). Development and implementation of the high-speed Schlieren technique was supported by the program (project VIU ENIN 94 2014) to improve the competitiveness of the National Research Tomsk Polytechnic University.

\section{References}

1. P. Stephan and C. Brandt, Heat Transfer Eng, 25 (3), 78 (2004)

2. V. Ajaev, Interfacial Phenomena and Heat Transfer,1 (1), 81 (2013)

3. O.A. Kabov, M.V. Bartashevich, V.V. Cheverda, Int. J. Emerging Multidiscip. Fluid Sci., 2 (2), $161(2010)$

4. O.A. Kabov, Yu.V. Lyulin, I.V. Marchuk, D.V. Zaitsev, International Journal of Heat and Fluid Flow, 28, 103 (2007) 